\title{
Convex Relaxations of Security Constrained AC Optimal Power Flow under Uncertainty
}

Venzke, Andreas; Chatzivasileiadis, Spyros

Published in:

Proceedings of 20th Power Systems Computation Conference

Link to article, DOI:

10.23919/PSCC.2018.8442940

Publication date:

2018

Document Version

Peer reviewed version

Link back to DTU Orbit

Citation (APA):

Venzke, A., \& Chatzivasileiadis, S. (2018). Convex Relaxations of Security Constrained AC Optimal Power Flow under Uncertainty. In Proceedings of 20th Power Systems Computation Conference IEEE.

https://doi.org/10.23919/PSCC.2018.8442940

\section{General rights}

Copyright and moral rights for the publications made accessible in the public portal are retained by the authors and/or other copyright owners and it is a condition of accessing publications that users recognise and abide by the legal requirements associated with these rights.

- Users may download and print one copy of any publication from the public portal for the purpose of private study or research.

- You may not further distribute the material or use it for any profit-making activity or commercial gain

- You may freely distribute the URL identifying the publication in the public portal

If you believe that this document breaches copyright please contact us providing details, and we will remove access to the work immediately and investigate your claim 


\title{
Convex Relaxations of Security Constrained AC Optimal Power Flow under Uncertainty
}

\author{
Andreas Venzke and Spyros Chatzivasileiadis \\ Department of Electrical Engineering \\ Technical University of Denmark \\ Kgs. Lyngby, Denmark \\ \{andven, spchatz\}@elektro.dtu.dk
}

\begin{abstract}
System operators have to ensure an N-1 secure operation, while dealing with higher degrees of uncertainty. This paper proposes a semidefinite relaxation of the chance and security constrained optimal power flow (SCOPF). Our main contributions are the introduction of systematic methods to obtain zero relaxation gap, providing a tractable chance constrained SCOPF formulation, and addressing scalability. We introduce a systematic procedure to obtain zero relaxation gap using a penalty term on power losses. To achieve tractability of the joint chance constraint, a piecewise affine approximation, and a combination of randomized and robust optimization is used. To address scalability, we propose an iterative solution algorithm to identify binding constraints, and we apply a chordal decomposition of the semidefinite constraints. We demonstrate the performance of our approach on IEEE 24 and IEEE 118 bus system using realistic day-ahead forecast data and obtain tight near-global optimality guarantees.
\end{abstract}

Index Terms-Chance constraints, contingency filtering, convex optimization, security constrained optimal power flow.

\section{INTRODUCTION}

To ensure power system security during steady-state operation, system operators require the fulfillment of the N-1 security criterion at all times. This stipulates that an outage of any single component or line does not lead to a violation of system constraints. Traditionally, the N-1 security criterion is evaluated by checking the determined operating set-point against a predefined set of critical contingencies. However, the increased integration of varying renewable generating sources and stochastic loads require tools that can determine optimal set-points considering both uncertainty and security constraints [1]. In this work, we propose a tractable convex formulation of the security constrained optimal power flow (SCOPF) under uncertainty, which models all AC power flow variables, includes corrective and preventive control policies related to active power, reactive power, and voltage, and can provide guarantees for global optimality. Our proposed method can be used by system operators to anticipate in day-ahead the impact of forecast errors on the N-1 security criterion.

The authors in [1] and [2] review the current state of the art of SCOPF formulations and identify the incorporation of uncertainty as a further development need. In the literature, to address the increasing uncertainty in power system operation, chance constraints which define a maximum allowable probability of constraint violation are included in SCOPF. Existing approaches [3]-[5] are limited to the DC-OPF approximation, which neglects losses, reactive power and voltage magnitudes. In the work [3], an analytical reformulation of a probabilistic security constrained DC-OPF is proposed which models the forecast errors of wind farms as Gaussian uncertainty distributions. This framework is extended in [4] to account for corrective control policies including HVDC and phaseshifting transformers. Making no prior assumptions on the uncertainty distributions, the work in [5] applies a combination of randomized and robust optimization to achieve a tractable formulation of the chance constraints. However, as the DCOPF formulation can exhibit substantial errors [6], the remainder of this paper will focus on the AC-OPF formulation.

To the authors' knowledge, the only works using an $\mathrm{AC}$ OPF formulation to address the SCOPF under uncertainty is [7]. This work uses the semidefinite relaxation of [8], and applies an affine approximation of the system state as a function of the forecast errors, and a combination of randomized and robust optimization to achieve tractability of the chance constraint. If the relaxation achieves zero relaxation gap, its solution corresponds to the global optimum of the non-convex AC-OPF with the affine approximation. However, in [7] the relaxation gap of the obtained solutions is not investigated.

In the literature, several security constrained AC-OPF formulations exist that do not consider uncertainty. For formulations related to the non-convex AC-OPF formulation, the interested reader can refer to references listed in [1]. For formulations related to the AC-OPF with convex relaxations, the work in [9] extends the semidefinite relaxation of [8] introducing security constraints and uses a loss penalty term on specific lines to achieve zero relaxation gap.

In our previous work [10], we introduced a comprehensive framework to handle chance constraints for a semidefinite formulation of the AC-OPF problem, including Gaussian distributions, and we investigated the relaxation gap. In this work, we introduce a security constrained OPF under uncertainty which uses the full AC-OPF formulation with convex relaxations and provides guarantees regarding the (near-)global optimality of the solution. Besides handling both security constraints and uncertainty in the original non-linear AC-OPF problem, the main contributions of this work are:

- We introduce a systematic procedure to obtain global optimality guarantees. By using a penalty term on power 
losses, we propose a method to identify suitable penalty terms for zero relaxation gap, i.e. we obtain solutions which are feasible to the original non-convex AC-OPF.

- Using a piecewise affine approximation of the system state as a function of the forecast errors, and applying a combination of randomized and robust optimization, we provide a tractable formulation of the chance constrained SCOPF. We include corrective and preventive control policies related to active power and voltage set-points of generators, and power factor set-points of wind farms.

- We propose two techniques to achieve scalability of our approach. First, we introduce a solution algorithm which iteratively determines binding constraints. In a filtering step, dominant contingencies and forecast errors in terms of maximum constraint violation magnitude are identified. Second, we propose a chordal decomposition of the semidefinite constraints.

- Using realistic forecast data, the performance of our proposed AC-SCOPF approach is evaluated on a IEEE 118 bus system and compared to the chance constrained DC-SCOPF of [5]. We find that our approach is more accurate and complies with the joint chance constraint whereas the DC-SCOPF violates both active generator limits and voltage limits. For the considered time steps, the near-global optimality guarantee is higher than $99.5 \%$.

This paper is structured as follows. Section II outlines the semidefinite relaxation of SCOPF and includes chance constraints. Section III proposes a tractable OPF formulation, and introduces a systematic method to obtain zero relaxation gap. Section IV addresses scalability. Section V presents results on the IEEE 24 and 118 bus test case. Section VI concludes.

\section{Optimal Power Flow Formulation}

\section{A. Semidefinite Relaxation of Security Constrained AC-OPF}

A power grid can be described as a graph which consists of buses $\mathcal{N}$ and lines $\mathcal{L}$. The set of generator buses is denoted with $\mathcal{G}$. To include security constraints, the set $\mathcal{C}$ contains the list of possible component outages. The first entry corresponds to the intact system state without any outage which is denoted by a 0 . The following auxiliary variables are introduced for each bus $k \in \mathcal{N}$, line $(l, m) \in \mathcal{L}$ and outage $c \in \mathcal{C}$ :

$$
\begin{aligned}
& Y_{k}^{c}:=e_{k} e_{k}^{T} Y^{c} \\
& Y_{l m}^{c}:=\left(\bar{y}_{l m}^{c}+y_{l m}^{c}\right) e_{l} e_{l}^{T}-\left(y_{l m}^{c}\right) e_{l} e_{m}^{T} \\
& \mathbf{Y}_{k}^{c}:=\frac{1}{2}\left[\begin{array}{ll}
\Re\left\{Y_{k}^{c}+\left(Y_{k}^{c}\right)^{T}\right\} & \Im\left\{\left(Y_{k}^{c}\right)^{T}-Y_{k}^{c}\right\} \\
\Im\left\{Y_{k}^{c}-\left(Y_{k}^{c}\right)^{T}\right\} & \Re\left\{Y_{k}^{c}+\left(Y_{k}^{c}\right)^{T}\right\}
\end{array}\right] \\
& \mathbf{Y}_{l m}^{c}:=\frac{1}{2}\left[\begin{array}{ll}
\Re\left\{Y_{l m}^{c}+\left(Y_{l m}^{c}\right)^{T}\right\} & \Im\left\{\left(Y_{l m}^{c}\right)^{T}-Y_{l m}^{c}\right\} \\
\Im\left\{Y_{l m}^{c}-\left(Y_{l m}^{c}\right)^{T}\right\} & \Re\left\{Y_{l m}^{c}+\left(Y_{l m}^{c}\right)^{T}\right\}
\end{array}\right] \\
& \overline{\mathbf{Y}}_{k}^{c}:=\frac{-1}{2}\left[\begin{array}{cc}
\Im\left\{Y_{k}^{c}+\left(Y_{k}^{c}\right)^{T}\right\} & \Re\left\{Y_{k}^{c}-\left(Y_{k}^{c}\right)^{T}\right\} \\
\Re\left\{\left(Y_{k}^{c}\right)^{T}-Y_{k}^{c}\right\} & \Im\left\{Y_{k}^{c}+\left(Y_{k}^{c}\right)^{T}\right\}
\end{array}\right] \\
& \overline{\mathbf{Y}}_{l m}^{c}:=\frac{-1}{2}\left[\begin{array}{cc}
\Im\left\{Y_{l m}^{c}+\left(Y_{l m}^{c}\right)^{T}\right\} & \Re\left\{Y_{l m}^{c}-\left(Y_{l m}^{c}\right)^{T}\right\} \\
\Re\left\{\left(Y_{l m}^{c}\right)^{T}-Y_{l m}^{c}\right\} & \Im\left\{Y_{l m}^{c}+\left(Y_{l m}^{c}\right)^{T}\right\}
\end{array}\right] \\
& M_{k}:=\left[\begin{array}{cc}
e_{k} e_{k}^{T} & 0 \\
0 & e_{k} e_{k}^{T}
\end{array}\right]
\end{aligned}
$$

For each outage $c \in \mathcal{C}$, matrix $Y^{c}$ denotes the bus admittance matrix of the power grid, $e_{k}$ the k-th basis vector, and $\bar{y}_{l m}^{c}$ the shunt admittance and $y_{l m}^{c}$ the series admittance of line $(l, m) \in \mathcal{L}$. Based on [9], the non-linear, non-convex $\mathrm{AC}$ SCOPF problem can be written using (1) - (7) as

$$
\begin{aligned}
\min _{W^{c}} \sum_{k \in \mathcal{G}}\left\{c_{k 2}\left(\operatorname{Tr}\left\{\mathbf{Y}_{k}^{0} W^{0}\right\}+P_{D_{k}}\right)^{2}+\right. \\
\left.c_{k 1}\left(\operatorname{Tr}\left\{\mathbf{Y}_{k}^{0} W^{0}\right\}+P_{D_{k}}\right)+c_{k 0}\right\}
\end{aligned}
$$

subject to the following constraints for each bus $k \in \mathcal{N}$, line $(l, m) \in \mathcal{L}$ and outage $c \in \mathcal{C}$ :

$$
\begin{array}{r}
\underline{P}_{G_{k}}^{c} \leq \operatorname{Tr}\left\{\mathbf{Y}_{k}^{c} W^{c}\right\}+P_{D_{k}} \leq \bar{P}_{G_{k}}^{c} \\
\underline{Q}_{G_{k}}^{c} \leq \operatorname{Tr}\left\{\overline{\mathbf{Y}}_{k}^{c} W^{c}\right\}+Q_{D_{k}} \leq \bar{Q}_{G_{k}}^{c} \\
\underline{V}_{k}^{2} \leq \operatorname{Tr}\left\{M_{k} W^{c}\right\} \leq \bar{V}_{k}^{2} \\
-\bar{P}_{l m}^{c} \leq \operatorname{Tr}\left\{\mathbf{Y}_{l m}^{c} W^{c}\right\} \leq \bar{P}_{l m}^{c} \\
\operatorname{Tr}\left\{\mathbf{Y}_{l m}^{c} W^{c}\right\}^{2}+\operatorname{Tr}\left\{\overline{\mathbf{Y}}_{l m}^{c} W^{c}\right\}^{2} \leq\left(\bar{S}_{l m}^{c}\right)^{2} \\
W^{c}=\left[\Re\left\{\mathbf{V}^{c}\right\} \Im\left\{\mathbf{V}^{c}\right\}\right]^{T}\left[\Re\left\{\mathbf{V}^{c}\right\} \Im\left\{\mathbf{V}^{c}\right\}\right]
\end{array}
$$

The objective (8) minimizes the generation cost of the intact system state. The terms $c_{k 2}, c_{k 1}$ and $c_{k 0}$ are the quadratic, linear and constant cost variables of generator $k \in \mathcal{G}$. The active and reactive power consumption at bus $k \in \mathcal{N}$ is defined in $P_{D_{k}}$ and $Q_{D_{k}}$. Constraints (9) and (10) include the nodal active and reactive power flow balances, and $\underline{P}_{G_{k}}^{c}$, $\bar{P}_{G_{k}}^{c}, \underline{Q}_{G_{k}}^{c}$ and $\bar{Q}_{G_{k}}^{c}$ are the generator limits for minimum and maximum active and reactive power for each outage $c \in \mathcal{C}$, respectively. The bus voltages are constrained by (11) with corresponding lower and upper limits $\underline{V}_{k}, \bar{V}_{k}$. The active and apparent power branch flow $P_{l m}^{c}$ and $S_{l m}^{c}$ on line $(l, m) \in \mathcal{L}$ are upper bounded by $\bar{P}_{l m}^{c}$ (12) and $\bar{S}_{l m}^{c}$ (13), respectively. This models emergency line ratings which allow a higher line loading for a defined period of time. Note, that each outage $c \in \mathcal{C}$ leads to a change of power flows and in turn to a change of complex bus voltages $\mathbf{V}^{c}$. In order to link the faulted system states $W^{c}$ with the intact system state $W^{0}$, preventive or post-contingency corrective control policies are included. This will be explained in detail in Section III-C. To obtain an optimization problem linear in $W$, the objective function is reformulated using Schur's complement:

$$
\begin{gathered}
\min _{\alpha, W^{c}} \sum_{k \in \mathcal{G}} \alpha_{k} \\
{\left[\begin{array}{cc}
c_{k 1} \operatorname{Tr}\left\{\mathbf{Y}_{k}^{0} W^{0}\right\}+a_{k} & \sqrt{c_{k 2} \operatorname{Tr}}\left\{\mathbf{Y}_{k}^{0} W^{0}\right\}+b_{k} \\
\sqrt{c_{k 2} \operatorname{Tr}}\left\{\mathbf{Y}_{k}^{0} W^{0}\right\}+b_{k} & -1
\end{array}\right] \preceq 0}
\end{gathered}
$$

where $a_{k}:=-\alpha_{k}+c_{k 0}+c_{k 1} P_{D_{k}}$ and $b_{k}:=\sqrt{c_{k 2}} P_{D_{k}}$. In addition, the apparent branch flow constraint (13) is rewritten:

$$
\left[\begin{array}{ccc}
-\left(\bar{S}_{l m}^{c}\right)^{2} & \operatorname{Tr}\left\{\mathbf{Y}_{l m}^{c} W^{c}\right\} & \operatorname{Tr}\left\{\overline{\mathbf{Y}}_{l m}^{c} W^{c}\right\} \\
\operatorname{Tr}\left\{\mathbf{Y}_{l m}^{c} W^{c}\right\} & -1 & 0 \\
\operatorname{Tr}\left\{\overline{\mathbf{Y}}_{l m}^{c} W^{c}\right\} & 0 & -1
\end{array}\right] \preceq 0
$$

The non-convex constraint (14) can be expressed by:

$$
\begin{aligned}
W^{c} & \succeq 0 \\
\operatorname{rank}\left(W^{c}\right) & =1
\end{aligned}
$$


The convex relaxation is introduced by dropping the rank constraint (19), relaxing the non-convex security constrained AC-OPF to a convex semidefinite program (SDP). The work in [9] proves that if the rank of all $W^{c}$ matrices obtained from the SDP relaxation is 1 or 2 , the obtained solution is the global optimum of the non-convex security constrained AC-OPF.

\section{B. Including Chance Constraints}

In power system operation, the shares of renewable electricity generation and of stochastic loads increase. To incorporate the associated forecast uncertainty, we extend the presented SCOPF formulation with a joint chance constraint [10]. A number of $n_{W}$ wind farms are introduced in the power grid at buses $k \in \mathcal{W}$ and the uncertain infeeds are modeled as

$$
P_{W_{k}}=P_{W_{k}}^{f}+\zeta_{k}
$$

where $P_{W}$ are the wind infeeds occurring in real-time, $P_{W}^{f}$ are the forecasted values and $\zeta$ are the forecast errors. The resulting SCOPF formulation includes the joint chance constraint for all buses $k \in \mathcal{N}$, lines $(l, m) \in \mathcal{L}$ and outages $c \in \mathcal{L}$ :

$$
\begin{aligned}
& \min _{\alpha, W^{c}} \sum_{k \in G} \alpha_{k} \\
& \text { s.t. } \underline{P}_{G_{k}}^{c} \leq \operatorname{Tr}\left\{\mathbf{Y}_{k}^{c} W^{c}\right\}+P_{D_{k}}-P_{W_{k}}^{f} \leq \bar{P}_{G_{k}}^{c} \text {, } \\
& \text { (10), (11), (12), (16), (17), (18) for } W^{c}=W_{0}^{c} \\
& \mathbb{P}\left\{\underline{P}_{G_{k}}^{c} \leq \operatorname{Tr}\left\{\mathbf{Y}_{k}^{c} W^{c}(\zeta)\right\}+P_{D_{k}}-P_{W_{k}}^{f}-\zeta_{k} \leq \bar{P}_{G_{k}}^{c},\right. \\
& \underline{Q}_{G_{k}}^{c} \leq \operatorname{Tr}\left\{\overline{\mathbf{Y}}_{k}^{c} W^{c}(\zeta)\right\}+Q_{D_{k}} \leq \bar{Q}_{G_{k}}^{c}, \\
& \underline{V}_{k}^{2} \leq \operatorname{Tr}\left\{M_{k} W^{c}(\zeta)\right\} \leq \bar{V}_{k}^{2}, \\
& -\bar{P}_{l m}^{c} \leq \operatorname{Tr}\left\{\mathbf{Y}_{l m}^{c} W^{c}(\zeta)\right\} \leq \bar{P}_{l m}^{c}, \\
& {\left[\begin{array}{ccc}
-\left(\bar{S}_{l m}^{c}\right)^{2} & \operatorname{Tr}\left\{\mathbf{Y}_{l m}^{c} W^{c}(\zeta)\right\} & \operatorname{Tr}\left\{\overline{\mathbf{Y}}_{l m}^{c} W^{c}(\zeta)\right\} \\
\operatorname{Tr}\left\{\mathbf{Y}_{l m}^{c} W^{c}(\zeta)\right\} & -1 & 0 \\
\operatorname{Tr}\left\{\overline{\mathbf{Y}}_{l m}^{c} W^{c}(\zeta)\right\} & 0 & -1
\end{array}\right] \preceq 0,} \\
& \left.W^{c}(\zeta) \succeq 0\right\} \geq 1-\epsilon
\end{aligned}
$$

For each outage $c \in \mathcal{C}$, the forecasted system state is denoted with $W_{0}^{c}$ and the function $W^{c}(\zeta)$ describes the changing system state as a function of the forecast errors. Both the constraints for the intact and faulted system states (23) - (28) should be satisfied with a joint probability higher than the parameter $(1-\epsilon) \in(0,1)$. The chance constrained SCOPF (21) - (28) is an infinite-dimensional problem optimizing over a set of matrices $W^{c}(\zeta)$ which are a function of a continuous uncertain variable $\zeta$. It is generally agreed that this renders the problem intractable and makes it necessary to identify a suitable approximation for $W^{c}(\zeta)$ [7].

\section{Tractable Optimal Power Flow Formulation}

Using a scenario-based method, we define the uncertainty set associated with the forecast errors. As the optimization problem is infinite-dimensional, we select a piecewise affine approximation of the system state as a function of the forecast errors. This allows us to include preventive and corrective control policies of generator active power and voltage setpoints, and of wind farm power factors. To achieve tractability
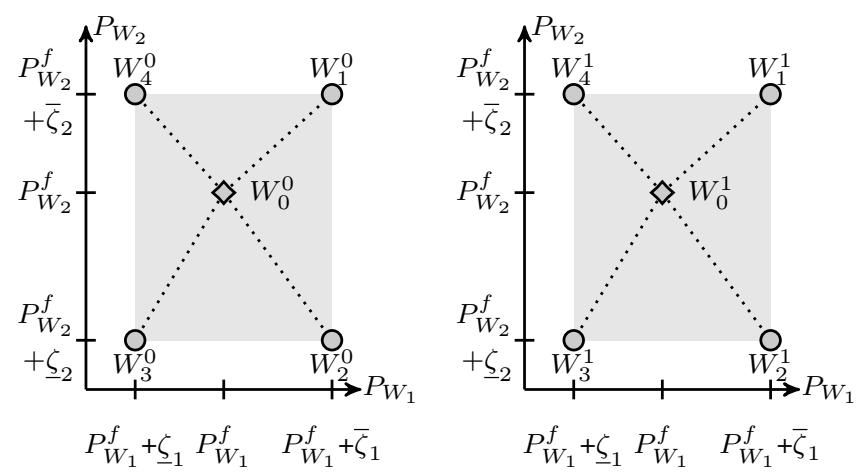

Figure 1. Rectangular uncertainty set displayed for two wind farms and one outage. The left figure corresponds to the intact system state and the right figure to the faulted system state. The piecewise affine approximation interpolates the system state between the forecasted system states $W_{0}^{0}$ and $W_{0}^{1}$ and the corresponding vertices of the uncertainty set $W_{1-4}^{0}$ and $W_{1-4}^{1}$ for the intact and the faulted system state, respectively.

of the resulting chance constraint, theoretical results from robust optimization are leveraged. By using a penalty term on power losses, we introduce a method to identify suitable penalty terms for zero relaxation gap.

\section{A. Scenario-Based Uncertainty Set}

To determine the bounds of the uncertainty set, we use a scenario-based method from [11], which does not make any assumption on the underlying distribution of the forecast errors. To this end, we compute the minimum volume rectangular set which with probability $1-\beta$ contains $1-\epsilon$ of the probability mass. The term $\beta$ is a confidence parameter. According to [7], it is necessary to draw at least the following number of scenarios $N_{\mathrm{s}}$ to specify the uncertainty set:

$$
N_{\mathrm{s}} \geq \frac{1}{1-\epsilon} \frac{e}{e-1}\left(\ln \frac{1}{\beta}+2 n_{W}-1\right)
$$

The term $e$ is Euler's number. The minimum and maximum bounds on the forecast errors $\zeta_{k} \in\left[\zeta_{k}, \bar{\zeta}_{k}\right]$ are retrieved by a simple sorting operation among the $N_{\mathrm{s}}$ scenarios. The vertices $v \in \mathcal{V}$ are the corner points of the rectangular uncertainty set. For each vertex $v \in \mathcal{V}$, the vector $\zeta_{v} \in \mathbb{R}^{n_{W}}$ contains the corresponding forecast error magnitudes of the wind farms.

\section{B. Piecewise Affine Approximation}

For the previously obtained rectangular uncertainty set, we use the piecewise affine approximation from [10] to model the system change as a function of the forecast errors. To this end, we introduce a matrix $W_{v}^{c}$ for each vertex $v \in \mathcal{V}$ and outage $c \in \mathcal{C}$. The system state as a function of the forecast errors is computed as a piece-wise affine interpolation between the forecasted system state $W_{0}^{c}$ and the vertices of the uncertainty set $W_{v}^{c}$ for each outage $c \in \mathcal{C}$ :

$$
W^{c}(\zeta)=W_{0}^{c}+\Psi_{v=1}^{n_{v}}(\zeta)\left(W_{v}^{c}-W_{0}^{c}\right)
$$

The function $\Psi_{v=1}^{n_{\mathrm{v}}}(\zeta)$ denotes a piecewise linear interpolation operator of the wind forecast error $\zeta$ between all vertices $\zeta_{v}$. It returns a weight for the direction of each vertex, corresponding to the distance. For the case of two uncertain wind infeeds and one considered outage, this concept is illustrated in Fig. 1. 


\section{Corrective and Preventive Control Policies}

In the control response, we differentiate between occurring forecast errors and line outages. We assume that the system operator can respond to forecast errors by including a corrective control policy for generator active power and voltage set-points, i.e. the system operator sends updated set-points based on the realization of forecast errors. As outages can happen instantaneously, we assume in this case that the setpoints remain fixed, that is we need to preventively anticipate the system change due to line outages.

During steady-state power system operation, generation has to match demand and system losses. If an imbalance occurs due to e.g. an occurring forecast error, designated generators in the power grid will respond by adjusting their active power output as part of automatic generation control (AGC). The vector $d_{G} \in \mathbb{R}^{n_{b}}$ defines the generator participation factors. The term $n_{b}$ denotes the number of buses. The vector $d_{W}^{i} \in \mathbb{R}^{n_{b}}$ has a $\{-1\}$ entry corresponding to the bus where the $i$-th wind farm is located at. The sum of the generator participation factors should compensate the deviation in wind generation, i. e. $\sum_{k \in G} d_{G_{k}}=1$. The line losses of the $\mathrm{AC}$ power grid vary non-linearly with changes in wind infeeds and changes in system topology. To allow for a compensation of the change in system losses according to the participation factors, we introduce a slack variable $\gamma_{v}^{c}$ for each vertex and outage. In order to link the generation dispatch of the forecasted system state $W_{0}^{0}$ with system states in which forecast errors occur, the following constraints are introduced for each vertex $v \in \mathcal{V} \backslash\{0\}$, outage $c \in \mathcal{C} \backslash\{0\}$ and wind feed-in $i$ in $\left\{1, n_{W}\right\}$ :

$$
\operatorname{Tr}\left\{\mathbf{Y}_{k}^{c} W_{v}^{c}-\mathbf{Y}_{k}^{0} W_{0}^{0}\right\}=\sum_{i}^{n_{W}} \zeta_{v_{i}}\left(d_{G_{k}}\left(1+\gamma_{v}^{c}\right)+d_{W_{k}}^{i}\right)
$$

As we assume that the generator active power set-points remain fixed in case of line outages, the following constraints should be included for each outage $c \in \mathcal{C} \backslash\{0\}$ :

$$
\operatorname{Tr}\left\{\mathbf{Y}_{k}^{c} W_{0}^{c}-\mathbf{Y}_{k}^{0} W_{0}^{0}\right\}=d_{G_{k}} \gamma_{0}^{c}
$$

We allow for a corrective control of voltage-set points at generator terminals in case of forecast errors. In case of outages, the generator voltage set-points remain fixed. Hence, the following constraints are included for each generator bus $k \in \mathcal{G}$, outage $c \in \mathcal{C}$ and vertex $v \in \mathcal{V}$ :

$$
\operatorname{Tr}\left\{M_{k} W_{v}^{c}\right\}=\operatorname{Tr}\left\{M_{k} W_{v}^{0}\right\}
$$

Grid codes specify reactive power capabilities of wind farms often in terms of power factor $\cos \phi$. We allow for a power factor set-point begin sent to each wind farm. Note, that our $\mathrm{AC}-\mathrm{OPF}$ framework captures the variation of the wind farm reactive power injection as a function of wind farm active power. To include the reactive power capabilities, we modify constraints (10) and (24) and we introduce the reactive power set-point $\tau_{k}$ for each wind farm $k \in \mathcal{W}$ :

$$
-\sqrt{\frac{1-\cos ^{2} \phi}{\cos ^{2} \phi}} \leq \tau_{k} \leq \sqrt{\frac{1-\cos ^{2} \phi}{\cos ^{2} \phi}}
$$

\section{Robust Optimization}

To obtain a tractable formulation of the chance constraint including the control policies, the following result from robust optimization is used: If the constraint functions are linear, monotone or convex with respect to the uncertain variables, then the system variables will only take the maximum values at the vertices of the uncertainty set [11]. Using the piecewise affine approximation of Section III-B, the constraints (23) (26) in the optimization problem are linear and the semidefinite constraints (27) and (28) are convex. Hence, it suffices to enforce the joint chance constraint at the vertices $v \in \mathcal{V}$ of the uncertainty set. We provide a tractable formulation of the joint chance constraint (23) - (28) for each vertex $v \in \mathcal{V}$, bus $k \in \mathcal{N}$, line $(l, m) \in \mathcal{L}$ and outage $c \in \mathcal{C}$ :

$$
\begin{aligned}
& \underline{P}_{k}^{c} \leq \operatorname{Tr}\left\{\mathbf{Y}_{k}^{c} W_{v}^{c}\right\}+P_{D_{k}}-P_{W_{k}}^{f}-\zeta_{v_{k}} \leq \bar{P}_{k}^{c} \\
& \underline{Q}_{k}^{c} \leq \operatorname{Tr}\left\{\overline{\mathbf{Y}}_{k}^{c} W_{v}^{c}\right\}+Q_{D_{k}}-\tau_{k}\left(P_{W_{k}}^{f}+\zeta_{v_{k}}\right) \leq \bar{Q}_{k}^{c} \\
& \underline{V}_{k}^{2} \leq \operatorname{Tr}\left\{M_{k} W_{v}^{c}\right\} \leq \bar{V}_{k}^{2} \\
& -\bar{P}_{l m} \leq \operatorname{Tr}\left\{\mathbf{Y}_{l m}^{c} W_{v}^{c}\right\} \leq \bar{P}_{l m} \\
& {\left[\begin{array}{ccc}
-\left(\bar{S}_{l m}\right)^{2} & \operatorname{Tr}\left\{\mathbf{Y}_{l m}^{c} W_{v}^{c}\right\} & \operatorname{Tr}\left\{\overline{\mathbf{Y}}_{l m}^{c} W_{v}^{c}\right\} \\
\operatorname{Tr}\left\{\mathbf{Y}_{l m}^{c} W_{v}^{c}\right\} & -1 & 0 \\
\operatorname{Tr}\left\{\overline{\mathbf{Y}}_{l m}^{c} W_{v}^{c}\right\} & 0 & -1
\end{array}\right] \preceq 0} \\
& W_{v}^{c} \succeq 0
\end{aligned}
$$

The chance constrained SCOPF formulation minimizes (21) subject to (22), (31) - (40) for each vertex $v \in \mathcal{V}$. For a detailed derivation in case outages are not considered, see [10].

\section{E. Systematic Procedure to Obtain Zero Relaxation Gap}

With relaxation gap, we refer to the gap between the semidefinite relaxation and a non-linear chance constrained SCOPF which uses the piecewise affine approximation to parametrize the solution space. To obtain zero relaxation gap for the chance constrained SCOPF, a loss penalty term is added to the objective function (21), where the terms $\mu_{v}^{c} \geq 0$ are weighting factors:

$$
\min _{\alpha, W_{v}^{c}, \gamma_{v}^{c}} \sum_{k \in \mathcal{G}} \alpha_{k}+\sum_{c \in \mathcal{C}} \sum_{v \in \mathcal{V}} \mu_{v}^{c} \gamma_{v}^{c}
$$

We use an individual penalty term $\mu_{v}^{c}$ for each vertex and outage instead of a uniform penalty parameter $\mu$ as in [10]. We found that this allows us to introduce a robust systematic method to obtain zero relaxation gap, i.e. identify rank1 solution matrices, as we will show in Section V-B. For this purpose, we solve the chance constrained SCOPF in an iterative manner. First, we set all penalty weights $\mu_{v}^{c}$ to 0 and solve the OPF problem. If we obtain rank-1 $W$ solutions, we terminate. Otherwise, we increase the penalty weight $\mu_{v}^{c}$ only for higher rank matrices by a defined step-size $\Delta \mu$ and resolve the OPF problem. We repeat this procedure until rank-1 solution matrices are identified. With this penalized semidefinite AC-OPF formulation, near-global optimality guarantees can be derived specifying the maximum distance to the global optimum [12]. The numerical results in Section V show that while this penalty is necessary to obtain zero relaxation gap, in practice the deviation from the global optimum is small. 


\section{SCALABILITY}

The size of the chance constrained SCOPF formulation in terms of number of $W$ matrices grows linearly with the number of outages and exponentially with the number of wind farms. To reduce computational complexity, we formulate a chordal decomposition of the semidefinite constraints and present an efficient iterative OPF solving strategy.

\section{A. Chordal Decomposition}

In the proposed OPF formulation the semidefinite constraint (40) is included for each considered vertex $v$ and outage $c$. To reduce the computational complexity, we formulate a chordal decomposition. Following [13], in order to obtain a chordal graph, a chordal extension of the power network is computed with a Cholesky factorization. Then, we compute the maximum cliques decomposition of the obtained chordal graph. We replace the semidefinite constraint (40) for each outage $c \in \mathcal{C}$, vertex $v \in \mathcal{V}$ with:

$$
\left(W_{v}^{c}\right)_{c l q, c l q} \succeq 0
$$

The positive semidefinite matrix completion theorem ensures that if (42) holds for each maximum clique $c l q$, the resulting matrix $W_{v}^{c}$ can be completed such that it is positive semidefinite. This allows to substantially reduce the number of considered matrix entries and the computational burden [13].

\section{B. Iterative Solution Algorithm}

We define a possible list of vertices $v$ and outages $c$ to be considered in the chance constrained SCOPF. We initialize the algorithm by solving the OPF problem without chance and security constraints, i.e. minimizing (21) subject to (22) for $W_{0}^{0}$, replacing (18) with (42). Then, we follow the iterative solution algorithm:

1) We run MATPOWER [14] AC power flows for each possible vertex and outage combination and evaluate the constraint violations. For the generator active power and voltage set-points, and wind farm power factor we consider the OPF control set-points. In case no constraint violations occur, we terminate the algorithm and have obtained a N-1 secure dispatch under uncertainty.

2) Otherwise, we apply a constraint filtering based on [15]. That is, we identify the vertex and outage combinations which for at least one constraint dominate all other combinations in terms of magnitude of constraint violation, i.e. for at least one specific constraint a dominant combination has the highest absolute constraint violation compared to all other combinations.

3) We add the identified dominating vertex and outage combinations in the OPF formulation by including (31) - (39) and (42) for each combination. We solve the resulting chance constrained SCOPF minimizing (41).

4) If we obtain rank-1 solutions for all included entries, we return to 1). Otherwise, we increase penalty weights for higher rank matrices by the defined step-size $\Delta \mu$ outlined in Section III-E, re-solve the OPF problem until rank-1 solution matrices are identified and return to 1 ).

\section{Simulation And Results}

We specify the simulation setup and apply the systematic procedure to obtain zero relaxation gap for an IEEE 24 bus system. We compare our proposed OPF formulation to a chance constrained DC-SCOPF for an IEEE 118 bus system.

\section{A. Simulation Setup}

The OPF is implemented in the optimization toolbox YALMIP [16] and the SDP solver MOSEK [17] is used. A small resistance of $10^{-4}$ per unit has to be added to each transformer, which is a condition for obtaining zero relaxation gap [8]. To investigate whether the rank of an obtained solution matrix $W$ is 2 , the ratio $\rho$ of the $2^{\text {nd }}$ to $3^{\text {rd }}$ eigenvalue is computed for each maximum clique $c l q$, a heuristic measure proposed by [18]. This value should be around $10^{5}$. Then, the respective rank-1 solution can be retrieved by following the procedure described in [18].

The work in [12] proposes the use of the following measure to evaluate the degree of the near-global optimality of a penalized SDP relaxation. Let $\tilde{f}_{1}(x)$ be the generation cost of the convex OPF without a penalty term and $\tilde{f}_{2}(x)$ the generation cost of the convex OPF with a penalty weight sufficiently high to obtain rank-1 solution matrices. Then, the near-global optimality can be assessed by computing the parameter $\delta_{\text {opt }}:=\frac{\tilde{f}_{1}(x)}{\tilde{f}_{2}(x)} \cdot 100 \%$. According to recent Grid Codes [19], we allow for a wind farm power factor ranging from 0.95 inductive to 0.95 capacitive. As emergency line rating $\bar{P}_{l m}^{c}$, we allow an increased short-term line limit of $120 \% \bar{P}_{l m}^{0}$. The confidence interval $1-\epsilon$ of the joint chance constraint is selected to be $95 \%$.

\section{B. Systematic Procedure to Obtain Zero Relaxation Gap}

In this section, we showcase the systematic procedure to obtain zero relaxation gap, i.e. to identify rank-1 solution matrices, using the IEEE 24 bus system from [14]. Three wind farms with a rated power of $150 \mathrm{MW}, 200 \mathrm{MW}$ and $200 \mathrm{MW}$ are placed at buses 3,10 and 20. For illustrative purposes, we assume for each wind farm a forecasted infeed of $50 \%$ of the rated power and assume the forecast error bounds are within $\pm 25 \%$ of the rated power with $95 \%$ probability. For the corrective control in case of forecast errors, each generator adjusts its active power proportional to its maximum active power. We include the outage of lines $\{2,9\}$. This results in $27 \mathrm{~W}$ matrices, corresponding to 8 vertices and 3 system states. We select the step penalty weight step size $\Delta \mu$ to be 20 and $10^{5}$ as minimum clique eigenvalue ratio. In Fig. 2 we compare the eigenvalue ratios for 40 iterations obtained from our proposed systematic procedure with uniformly increasing the penalty weight on all matrices as in [10]. In this case, we find that the latter does not obtain zero relaxation gap, i.e. rank-1 solution matrices, whereas our proposed systematic procedure allows us to obtain zero relaxation gap at iteration 30. At this point, the near-global optimality guarantee evaluates to $99.77 \%$, i.e. the distance to the global optimum is at most $0.23 \%$. The proposed procedure allows for a systematic identification of 


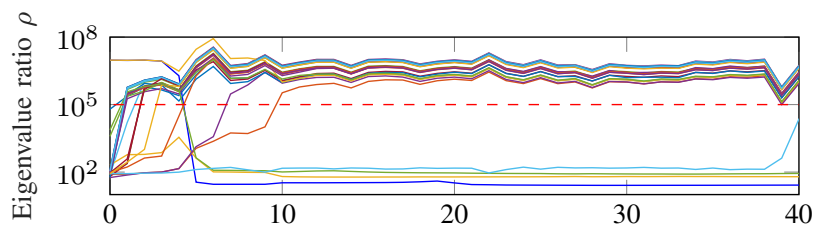

(a) - Number of iterations for uniform penalty term

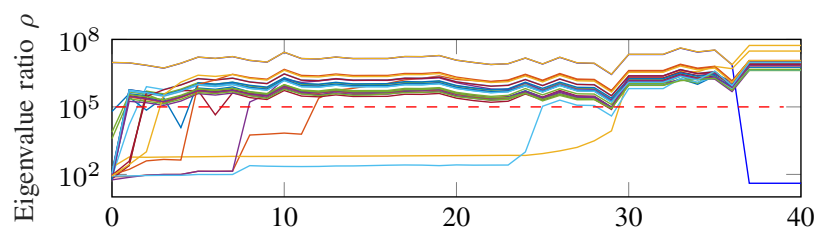

(b) - Number of iterations for systematic penalty term

Figure 2. The eigenvalue ratios $\rho$ for each iteration of the (b) systematic procedure are compared to the (a) uniform penalty parameter of [10] for a IEEE 24 bus test case with three wind farms and two considered line outages. The systematic procedure would terminate at iteration 30 . In this test case only, we extend the number of iterations to 40 to further investigate the relationship between eigenvalue ratio and penalty parameter. For this purpose, once we reach the defined minimum clique eigenvalue ratio, we double it.

suitable penalty weights. This improves upon previous works [9], [10] which use an ad-hoc defined penalty parameter. In case simulations with similar setup are rerun, the previously obtained penalty weights $\mu_{v}^{c}$ can be used as a hot start.

\section{IEEE 118 Bus Test Case}

In this section, we compare the chance constrained ACSCOPF from Section IV-B (CC-AC-SCOPF) with a chance constrained DC-SCOPF formulation (CC-DC-SCOPF) from [5]. The DC-SCOPF formulation includes a joint chance constraint on active power and active line flows and uses the combination of randomized and robust optimization to achieve a tractable chance constraint formulation. We use the IEEE 118 bus test case from [20] with the following modifications. Voltage upper and lower bounds are assumed to be 1.1 per unit and 0.9 per unit. To obtain a more constrained system, we increase the active and reactive load demand by $30 \%$. Note that the line limits are defined in terms of active power. We include 3 wind farms at the buses 5, 37 and 60 with a rated power of $300 \mathrm{MW}, 600 \mathrm{MW}$ and $400 \mathrm{MW}$, respectively. The total rated wind power corresponds to $24.7 \%$ of total load demand. We assume a confidence parameter $\beta=10^{-3}$. To construct the rectangular uncertainty set for both formulations, we draw $N_{S}=377$ samples from realistic day-ahead wind forecast scenarios from [21]. The forecasts are based on wind power measurements in the Western Denmark area from 15 different control zones collected by the Danish transmission system operator Energinet. We select control zone 2, 4, 10 to correspond to the wind farm at bus 5,37 , and 60 , respectively. The forecast data and forecast error bounds for the 7 considered time steps are shown in Fig. 3. We consider the following list of outages of lines: $\mathcal{C}=\{0,6,20,35,99,111,136,138,155,164,172,175\}$. For corrective control in case of forecast errors, all generation units with an upper active power limit larger than $100 \mathrm{MW}$ are assigned a participation factor proportional to their maximum

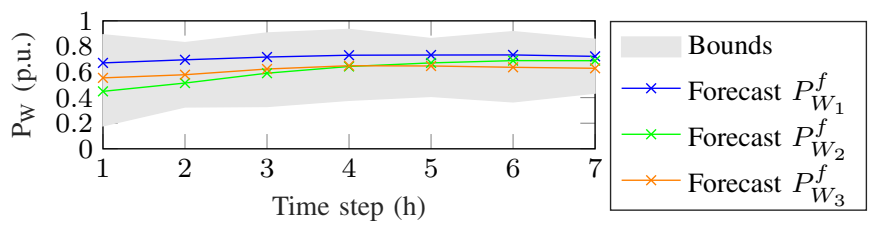

Figure 3. Wind forecast from hour 1 to hour 7: The bounds correspond to the overall minimum and maximum values from the $N_{s}$ sampled scenarios.

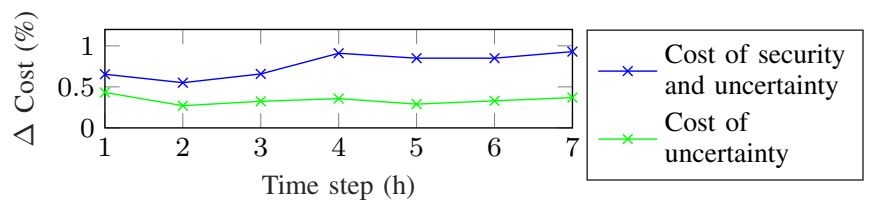

Figure 4. Cost of security and uncertainty and cost of uncertainty: $100 \%$ corresponds to generation cost without consideration of security and uncertainty.

power limit. Due to the numerical accuracy of SDP solvers, we define $0.5 \times 10^{5}$ as minimum clique eigenvalue ratio for zero relaxation gap. A penalty step size $\Delta \mu$ of 25 is used.

In Fig. 4, the relative cost increase for the chance constrained AC-SCOPF compared to an AC-OPF without consideration of security and uncertainty is shown. Furthermore, we show the cost of uncertainty, i.e. the cost increase when security constraints are not considered. We find for time step 7 that the cost of security and uncertainty is $0.93 \%$ whereas the cost of uncertainty alone is $0.37 \%$, i.e. the generation cost increases by including security constraints. The chance constrained DC-SCOPF evaluates to a lower generation cost compared to the AC-OPF as power losses are not considered.

We run a Monte Carlo Analysis using MATPOWER AC power flows with 5 '000 samples drawn from the realistic forecast data to evaluate the constraint violation for all contingencies $\mathcal{C}$. To exclude numerical errors, a minimum violation limit of $10^{-3}$ per unit for generator limits on active power and $0.1 \%$ for voltage and active line flow limits is assumed. In the $\mathrm{AC}$ power flow the generator reactive power limits are enforced to avoid a possibly high non-physical overloading of the limits [22]. In Table I the resulting violation probabilities of the system constraints on active power, bus voltages, and active

TABLE I

COMPARISON OF CONSTRAINT VIOLATION PROBABILITY FOR OUR PROPOSED METHOD AND THE CC-DC-SCOPF [5] FOR 5'000 REALISTIC FORECAST DATA SAMPLES. INSECURE INSTANCES MARKED WITH BOLD.

\begin{tabular}{lccccccc}
\hline \hline Time step (h) & 1 & 2 & 3 & 4 & 5 & 6 & 7 \\
\hline \hline \multicolumn{7}{c}{ Bus voltages $(\%)$} \\
\hline CC-AC-SCOPF & 0.0 & 0.0 & 0.0 & 0.0 & 0.0 & 0.0 & 0.0 \\
\hline CC-DC-SCOPF & $\mathbf{9 3 . 8}$ & $\mathbf{1 0 0}$ & $\mathbf{9 9 . 8}$ & $\mathbf{9 9 . 2}$ & $\mathbf{9 9 . 9}$ & $\mathbf{9 8 . 3}$ & $\mathbf{1 0 0}$ \\
\hline \hline \multicolumn{7}{c}{ Active power line limits (\%) } \\
\hline CC-AC-SCOPF & 0.0 & 0.0 & 0.0 & 0.0 & 0.0 & 0.0 & 0.0 \\
\hline CC-DC-SCOPF & 0.0 & 0.0 & 0.0 & 0.0 & 0.0 & 0.0 & 0.0 \\
\hline \hline \multicolumn{7}{c}{ Active generator limits (\%) } \\
\hline CC-AC-SCOPF & 0.0 & 0.0 & 0.7 & 0.0 & 0.0 & 0.8 & 0.0 \\
\hline CC-DC-SCOPF & $\mathbf{1 4 . 4}$ & $\mathbf{1 3 . 7}$ & $\mathbf{1 6 . 9}$ & $\mathbf{2 4 . 7}$ & $\mathbf{3 4 . 0}$ & $\mathbf{5 2 . 8}$ & $\mathbf{2 5 . 5}$ \\
\hline \hline
\end{tabular}


TABLE II

NEAR-GLOBAL OPTIMALITY GUARANTEES, SHARE OF BINDING VERTEX AND OUTAGE COMBINATIONS, AND COMPUTATIONAL TIME

\begin{tabular}{ccccccc}
\hline \hline \multicolumn{7}{c}{ Time step (h) } \\
\hline 1 & 2 & 3 & 4 & 5 & 6 & 7 \\
\hline \hline \multicolumn{7}{c}{ Near-global optimality guarantees $\delta_{\text {opt }}(\%)$} \\
\hline 99.95 & 99.92 & 99.91 & 99.71 & 99.72 & 99.76 & 99.72 \\
\hline \hline \multicolumn{7}{c}{ Share of binding vertex and outage combinations $(\%)$} \\
\hline 6.5 & 6.5 & 8.3 & 9.3 & 9.3 & 8.3 & 7.4 \\
\hline \hline & Total SDP solver solution time (s) \\
\hline 24.1 & 17.8 & 60.8 & 156.9 & 171.0 & 116.0 & 101.8 \\
\hline \hline
\end{tabular}

branch flows are compared for our approach and the chance constrained DC-SCOPF. We find that our proposed approach complies with the joint chance constraint violation probability of $5 \%$. The DC-SCOPF violates both voltage and active power generator limits. The voltage violations occur as the DC-OPF approximation does not model voltage magnitudes. As losses are neglected, the limits on generator active power are violated.

For the considered time steps, Table II shows that we obtain tight near-global optimality guarantees higher than $99.5 \%$. The iterative solution algorithm allows us to reduce the number of considered vertex and outage combinations in step 3) in Section IV-B to less than 10\%. In our implementation, the 118 bus test case is intractable without applying both the chordal decomposition and the iterative solution algorithm. Using a laptop with Intel i7-7820HQ CPU @ $2.90 \mathrm{GHz}$ and $32 \mathrm{~GB}$ RAM, the run-time of the overall iterative solution algorithm is on average 6 minutes out of which 1.5 minutes are spend solving the SDP. In future work, we aim to develop a more efficient implementation and parallelize the computation by means of distributed optimization techniques.

\section{Conclusions}

In this work, we propose a chance constrained and security constrained AC-OPF. Using a piecewise affine approximation, we can evaluate near-global optimality, and include control policies related to active power, reactive power, and voltage. With a penalty term on losses, we introduce a systematic procedure to obtain zero relaxation gap. For a IEEE 118 bus test case using realistic forecast data, we show that a chance constrained DC-SCOPF leads to violations of both active power and voltage limits whereas our proposed approach complies with the joint chance constraint violation probability. Notably, our approach obtains tight near-global optimality guarantees specifying the distance to the global optimum to be upper bounded by $0.5 \%$ of the objective value. To address scalability, we propose an iterative solution algorithm which reduces the problem size in this test case by $90 \%$. Our future work will focus on including (i) HVDC grids, (ii) Gaussian distributions and (iii) distributed optimization.

\section{REFERENCES}

[1] F. Capitanescu, J. M. Ramos, P. Panciatici, D. Kirschen, A. M. Marcolini, L. Platbrood, and L. Wehenkel, "State-of-the-art, challenges, and future trends in security constrained optimal power flow," Electric Power Systems Research, vol. 81, no. 8, pp. 1731-1741, 2011.

[2] F. Capitanescu, "Critical review of recent advances and further developments needed in AC optimal power flow," Electric Power Systems Research, vol. 136, pp. 57-68, 2016.

[3] L. Roald, F. Oldewurtel, T. Krause, and G. Andersson, "Analytical reformulation of security constrained optimal power flow with probabilistic constraints," in IEEE PowerTech, Grenoble, France, 2012.

[4] L. Roald, S. Misra, T. Krause, and G. Andersson, "Corrective control to handle forecast uncertainty: A chance constrained optimal power flow," IEEE Transactions on Power Systems, vol. 32, no. 2, pp. 1626-1637, 2017.

[5] M. Vrakopoulou, K. Margellos, J. Lygeros, and G. Andersson, "A probabilistic framework for reserve scheduling and N-1 security assessment of systems with high wind power penetration," IEEE Transactions on Power Systems, vol. 28, no. 4, pp. 3885-3896, 2013.

[6] K. Dvijotham and D. K. Molzahn, "Error bounds on the DC power flow approximation: A convex relaxation approach," in 2016 IEEE 55th Conference on Decision and Control (CDC), 2016, pp. 2411-2418.

[7] M. Vrakopoulou, M. Katsampani, K. Margellos, J. Lygeros, and G. Andersson, "Probabilistic security-constrained AC optimal power flow," in IEEE PowerTech, Grenoble, France, 2012.

[8] J. Lavaei and S. H. Low, "Zero duality gap in optimal power flow problem," IEEE Transactions on Power Systems, vol. 27, no. 1, pp. 92-107, 2012.

[9] R. Madani, M. Ashraphijuo, and J. Lavaei, "Promises of conic relaxation for contingency-constrained optimal power flow problem," IEEE Transactions on Power Systems, vol. 31, no. 2, pp. 1297-1307, 2016.

[10] A. Venzke, L. Halilbasic, U. Markovic, G. Hug, and S. Chatzivasileiadis, "Convex relaxations of chance constrained AC optimal power flow," IEEE Transactions on Power Systems, vol. PP, no. 99, pp. 1-1, 2017.

[11] K. Margellos, P. Goulart, and J. Lygeros, "On the road between robust optimization and the scenario approach for chance constrained optimization problems," IEEE Transactions on Automatic Control, vol. 59, no. 8, pp. 2258-2263, 2014.

[12] R. Madani, S. Sojoudi, and J. Lavaei, "Convex relaxation for optimal power flow problem: Mesh networks," IEEE Transactions on Power Systems, vol. 30, no. 1, pp. 199-211, 2015.

[13] R. A. Jabr, "Exploiting sparsity in SDP relaxations of the OPF problem," IEEE Transactions on Power Systems, vol. 27, no. 2, pp. 1138-1139, 2012.

[14] R. D. Zimmerman, C. E. Murillo-Sánchez, and R. J. Thomas, "MATPOWER: Steady-state operations, planning, and analysis tools for power systems research and education," IEEE Transactions on Power Systems, vol. 26, no. 1, pp. 12-19, 2011.

[15] F. Capitanescu, M. Glavic, D. Ernst, and L. Wehenkel, "Contingency filtering techniques for preventive security-constrained optimal power flow," IEEE Transactions on Power Systems, vol. 22, no. 4, pp. 1690$1697,2007$.

[16] J. Löfberg, "YALMIP : A toolbox for modeling and optimization in MATLAB," in In Proceedings of the CACSD Conference, Taipei, Taiwan, 2004.

[17] MOSEK ApS, Release 8.1.0.24., 2017. [Online]. Available: http: //docs.mosek.com/8.1/toolbox.pdf

[18] D. K. Molzahn, J. T. Holzer, B. C. Lesieutre, and C. L. DeMarco, "Implementation of a large-scale optimal power flow solver based on semidefinite programming," IEEE Transactions on Power Systems, vol. 28, no. 4, pp. 3987-3998, 2013.

[19] M. Tsili and S. Papathanassiou, "A review of grid code technical requirements for wind farms," IET Renewable Power Generation, vol. 3, no. 3, pp. 308-332, 2009.

[20] "IEEE 118-bus, 54-unit, 24-hour system,", Electrical and Computer Engineering Department, Illinois Institute of Technology, Tech. Rep. [Online]. Available: http://motor.ece.iit.edu/data/JEAS_IEEE118.doc

[21] W. A. Bukhsh, C. Zhang, and P. Pinson, "An integrated multiperiod OPF model with demand response and renewable generation uncertainty," IEEE Transactions on Smart Grid, vol. 7, no. 3, pp. 1495-1503, 2016.

[22] H. Vu, P. Pruvot, C. Launay, and Y. Harmand, "An improved voltage control on large-scale power system," IEEE Transactions on Power Systems, vol. 11, no. 3, pp. 1295-1303, 1996. 